\title{
Stories of both agency and acceptability were identified in accounts of HIV infected individuals having unprotected sex
}

Rhodes T, Cusick L. Accounting for unprotected sex: stories of agency and acceptability. Soc Sci Med 2002;55:211-26.

\section{QUESTION: How do HIV infected individuals account for themselves and their behaviour in situations where unprotected sex has occurred?}

Design

Indepth interviews.

\section{Setting}

London area, UK.

\section{Participants}

61 people living with HIV infection and 12 sexual partners of people living with HIV infection were recruited using a purposive sampling strategy. The sample comprised 22 self described homosexuals, 42 heterosexuals, and 9 bisexuals. The sample also included drug users who had never injected, former and current injecting drug users, regular and infrequent users, and 3 never drug users.

\section{Methods}

Data were collected during 1-2 hour, loosely structured, indepth interviews conducted at HIV or drug support agencies, homes of interviewees, or the research department. Interview data were audiotaped, transcribed, coded, and analysed inductively. A questionnaire was used to collect demographic data including HIV testing, diagnosis, drug use, and recent sexual history.

\section{Main findings}

2 types of accounts were identified: stories of agency and stories of acceptability. Stories of agency were those in which participants presented accounts to excuse themselves from personal responsibility associated with risky sexual activities. These "excuse type" accounts appeal to the notion of "defeasibility" and consist of 2 dimensions: (1) obstacles to applying knowledge of risk associated with having unprotected sex, and (2) a lack of power to translate risk knowledge and good intentions into actual risk avoidance. Obstacles were often cited historically and included a lack of knowledge about HIV transmission and errors in risk calculation based on poor information and factors beyond their control, such as condom failure. Other key categories of appeal to defeasibility included the influence of alcohol and drugs, powerlessness or coercion, and forces of nature, all of which absolved the individual for unprotected sex.

Stories of acceptability placed the participant in resistance to popular perceptions of risk rationality, acceptability, and expertise. Unprotected sex was justified as an acceptable risk along the themes of (1) antibody concordance, (2) relationship commitment and emotion, and (3) alter responsibility. Negotiated acceptance and minimal risk rationalisation regarding unprotected sex between HIV positive partners underpinned antibody concordance accounts. Relationship security and commitment justified alternative risk acceptability of unprotected sex, contrary to public health doctrine. Appeals to alter responsibility were identified in persons with HIV infection, who were only responsible for their own safety and not that of their partners, given that their positive status and suggestions for using protection were clearly tabled. In a rare account, intentional HIV transmission was justified in terms of the victim being deserving of injury in direct response to injury received.

\section{Conclusion}

Stories of agency and acceptability were identified in accounts of HIV infected individuals having unprotected sex.

\section{COMMENTARY}

Rhodes and Cusick provide narratives that describe expected and unexpected rationale for engaging in unprotected sex by people who are HIV positive. The study findings are similar to other studies accounting for the expected reasons people engage in unprotected sex, but adds a layer of thought about unexpected reasons called "post-modern fatalism".

The sample of participants was unusual in size and composition. The large number of participants $(n=75)$ is more commonly seen in studies that attempt to prove causality rather than explicate participants' stories. Quotas for diverse categories of people who were HIV positive and sexual partners of those who were HIV positive were obtained. This combination of a large sample and the achievement of quotas for specific categories suggests transferability of the findings beyond the study sample. However, the status of drug use during interviews raises questions of data integrity. More importantly, I question if qualitative sampling was actually achieved via theoretical saturation (confirmation of information obtained) and redundancy (no new news) in this study. ${ }^{1}$ Another concern is that 66 of the interviews were completed by the authors, and 7 were completed by a research student. In qualitative studies, the researcher is the instrument. ${ }^{2}$ Burns and Grove state that "... the researcher influences the individuals being studied and, in turn, is influenced by them". ${ }^{3}$ As such, were there substantial differences between the interviews done by the authors compared with those done by the research student?

The study by Rhodes and Cusick provides numerous examples of how and why people who are HIV positive engage in unprotected sex. Awareness of these issues enhances the ability of nurses and other health personnel to assist those who are HIV positive and their partners in intimate relationship negotiation. Further study is recommended to ascertain the transferability of the findings of this study to other contexts and other people who are HIV positive.

Nancy Nightingale Gillespie, RN, PhD Professor and Chair, Department of Nursing University of Saint Francis Fort Wayne, Indiana, USA

1 Denzin NK, Lincoln YS. Handbook of qualitative inquiry. Thousand Oaks: Sage Publications, 1994.

Lincoln YS, Guba EG. Naturalistic inquiry. Beverly Hills, CA: Sage Publications, 1985.

Burns N, Grove SK. The practice of nursing research:conduct, critique, and utilization. Philadelphia: WB Saunders, 2001. 